\title{
Correction to: Factors associated with anxiety and depression in rheumatoid arthritis patients: a cross-sectional study
}

\author{
Miyabi Uda ${ }^{1}$ (D, Motomu Hashimoto ${ }^{2,3}$, Ryuji Uozumi ${ }^{4}$, Mie Torii ${ }^{1}$, Takao Fujii ${ }^{5}$, Masao Tanaka², Moritoshi Furu², \\ Hiromu Ito ${ }^{2}$, Chikashi Terao ${ }^{6,7,8,9}$, Wataru Yamamoto ${ }^{10}$, Genichi Sugihara ${ }^{11}$, Yukako Nakagami ${ }^{11,12}$, \\ Tsuneyo Mimori ${ }^{13,14}$ and Kazuko $\mathrm{Nin}^{1 *}$ (i)
}

\section{Correction to: Adv Rheumatol (2021) 61:65} https://doi.org/10.1186/s42358-021-00223-2

Following publication of the original article [1], the authors would like to correct several errors in the article body.

\section{In the Abstract, Methods section:}

The first sentence should read: We conducted a crosssectional study of RA patients from the Kyoto University Rheumatoid Arthritis Management Alliance cohort.

In the Abstract, Results:

The incorrect sentence reads:

Additionally, HAQ-DI scores $\leq 0.5$ were associated with anxiety symptoms (OR [95\% CI] 3.51 [1.85-6.64]; $\mathrm{p}<0.01)$ and depression symptoms (OR [95\% CI] 2.65 [1.56-4.50]; $\mathrm{p}<0.01)$.

The sentence should read:

Additionally, HAQ-DI scores $>0.5$ were associated with anxiety symptoms (OR [95\% CI] 3.51 [1.85-6.64]; $\mathrm{p}<0.01)$ and depression symptoms (OR [95\% CI] 2.65 [1.56-4.50]; $\mathrm{p}<0.01)$.

The original article can be found online at https://doi.org/10.1186/s42358021-00223-2.

\footnotetext{
*Correspondence: nin@kuhp.kyoto-u.ac.jp

${ }^{1}$ Department of Human Health Sciences, Kyoto University Graduate

School of Medicine, 53 Shogoin Kawahara-cho, Sakyo-ku, Kyoto 606-8507, Japan

Full list of author information is available at the end of the article
}

In the Abstract, Conclusions:

The incorrect sentence reads:

Patients with high PtGA scores or HAQ-DI scores $\leq$ 0.5 were more likely to experience anxiety and depression symptoms, irrespective of disease activity remission status.

The sentence should read:

Patients with high PtGA scores or HAQ-DI-based nonremission were more likely to experience anxiety and depression symptoms, irrespective of disease activity remission status.

In the Methods section, Patient and setting sub-section:

The first sentence should read: We performed a crosssectional analysis of patients who visited the outpatient RA center of Kyoto University Hospital between May 1 and December 31, 2014, and whose data were collected in the 2014 Kyoto University Rheumatoid Arthritis Management Alliance (KURAMA) cohort [29].

The last sentence should read: The study protocol was approved by the Kyoto University Graduate School and Faculty of Medicine, Ethics Committee, and all procedures were performed in accordance with the 1964 Declaration of Helsinki and its later amendments or comparable ethical standards.

\section{In the Funding section:}

The sentence should read: The KURAMA cohort study is supported by a grant from Daiichi Sankyo Co., Ltd. 


\section{In the Ethics Approval and Consent to Participate section:}

The sentence should read: The study protocol was approved by the Kyoto University Graduate School and Faculty of Medicine, Ethics Committee, and all procedures were performed in accordance with the 1964 Declaration of Helsinki and its later amendments, or comparable ethical standards.

The authors apologize the inconvenience caused.

\begin{abstract}
Author details
${ }^{1}$ Department of Human Health Sciences, Kyoto University Graduate School of Medicine, 53 Shogoin Kawahara-cho, Sakyo-ku, Kyoto 606-8507, Japan. ${ }^{2}$ Department of Advanced Medicine for Rheumatic Diseases, Kyoto University Graduate School of Medicine, Kyoto, Japan. ${ }^{3}$ Department of Clinical Immunology, Graduate School of Medicine, Osaka City University, Osaka, Japan. ${ }^{4}$ Department of Biomedical and Bioinformatics, Kyoto University Graduate School of Medicine, Kyoto, Japan. ${ }^{5}$ Department of Rheumatology and Clinical Immunology, Wakayama Medical University, Wakayama, Japan. ${ }^{6}$ Department of Rheumatology and Clinical Immunology, Graduate School of Medicine, Kyoto University, Kyoto, Japan. ${ }^{7}$ Laboratory for Statistical and Translational Genetics, RIKEN Center for Integrative Medical Sciences, Yokohama, Japan. ${ }^{8}$ Clinical Research Center, Shizuoka General Hospital, Shizuoka, Japan. ${ }^{9}$ The Department of Applied Genetics, The School of Pharmaceutical Sciences, University of Shizuoka, Shizuoka, Japan. ${ }^{10}$ Department of Health Information Management, Kurashiki Sweet Hospital, Okayama, Japan. ${ }^{11}$ Department of Psychiatry, Kyoto University Graduate School of Medicine, Kyoto, Japan. ${ }^{12}$ Kyoto University Health Service, Kyoto, Japan. ${ }^{13}$ Department of Rheumatology and Clinical Immunology, Kyoto University Graduate School of Medicine, Kyoto, Japan. ${ }^{14}$ Ijinkai Takeda General Hospital, Kyoto, Japan.
\end{abstract}

Published online: 02 December 2021

\section{Reference}

1. Uda et al. Adv Rheumatol (2021) 61:65 https://doi.org/10.1186/ s42358-021-00223-2

\section{Publisher's Note}

Springer Nature remains neutral with regard to jurisdictional claims in published maps and institutional affiliations.
Ready to submit your research? Choose BMC and benefit from:

- fast, convenient online submission

- thorough peer review by experienced researchers in your field

- rapid publication on acceptance

- support for research data, including large and complex data types

- gold Open Access which fosters wider collaboration and increased citations

- maximum visibility for your research: over 100M website views per year

At BMC, research is always in progress.

Learn more biomedcentral.com/submissions 